\title{
Analysis of EPONs Under the Static Priority Scheduling Scheme with Fixed Transmission Times
}

\author{
Holmberg, Torgny
}

Published in:

Proceedings of 2nd Conference on Next Generation Internet Design and Engineering

Link to article, DOI:

10.1109/NGI.2006.1678241

Publication date:

2006

Document Version

Publisher's PDF, also known as Version of record

Link back to DTU Orbit

Citation (APA):

Holmberg, T. (2006). Analysis of EPONs Under the Static Priority Scheduling Scheme with Fixed Transmission Times. In Proceedings of 2nd Conference on Next Generation Internet Design and Engineering (pp. 199-206). IEEE. https://doi.org/10.1109/NGI.2006.1678241

\section{General rights}

Copyright and moral rights for the publications made accessible in the public portal are retained by the authors and/or other copyright owners and it is a condition of accessing publications that users recognise and abide by the legal requirements associated with these rights.

- Users may download and print one copy of any publication from the public portal for the purpose of private study or research.

- You may not further distribute the material or use it for any profit-making activity or commercial gain

- You may freely distribute the URL identifying the publication in the public portal 


\title{
Analysis of EPONs Under the Static Priority Scheduling Scheme with Fixed Transmission Times
}

\author{
Torgny Holmberg \\ Research Center COM \\ Technical University of Denmark \\ Building 345V, DK-2800 Kgs. Lyngby, Denmark. \\ E-mail: th@com.dtu.dk.
}

\begin{abstract}
In this paper, we analyse the Ethernet Passive Optical Network (EPON) and formulate dimensioning problems. Under the assumption that the transmission times are fixed in duration and that the input traffic is rate limited and scheduled by the Static Priority scheme, we provide means for dimensioning the network in order to keep the required hard deadlines. Expressions of the frame delay are derived as functions of the arrival curves of the different traffic sources. Furthermore, the arrival regulator structure is general and the only requirements are that the arrival process is causal and that the regulator provides sub-additivity.
\end{abstract}

In this study, we find that the examined structure is highly inefficient with poor utilisation and where the deadlines basically is provided by means of overdimensioning. The static structure of the bandwidth allocation scheme allows no adjustment of the current traffic load and must therefore proactively allocate resources yielding a rigid and inefficient network.

\section{INTRODUCTION}

Recent years rapid increase of high data rate equipments such as Gigabit Ethernet together with the increasing population of users with network capacity demanding application, have sped up the need for development and deployment of high capacity access networks. Today's copper based xDSL access networks are now regarded as insufficient and the search light has been put on the optical fiber, i.e., solutions such as fiber-to-thecurb (FTTC) or fiber-to-the-home (FTTH). The access network capacity is thus highly increased though it potentially is a far more expensive solution. However, by exchanging active network elements in favor of the cheaper passive elements, e.g., splitters and couplers, cost can be cut drastically. The Ethernet Passive Optical Network (EPON) is one such solution [1]. It adopts a point-to-multi point topology of either tree, ring or bus structure though the tree topology is the most common. With the tree topology, the structure applied in this paper, the root provides the connection to the Metropolitan Area
Networks (MANs) or Wide Area Networks (WANs), and the leafs provide the user sites the network access. As the name implies, Ethernet data link frames encapsulate the higher layer data enabling easy interoperability between the user networks and the access network as the Ethernet Local Area Network (LAN) is the most common LAN type. Furthermore, EPON networks are intended to implement the same standard data rates as the traditional non-optical Ethernet networks. A single trunk fiber connects the head-end with the subscriber nodes via a passive optical splitter. Only two wavelengths, one in each direction, are used for sending the information and thus only a single transceiver is used in the headend. This structure implies transmission broadcasting and time division multiplexing, where in EPON, the devices on the user side request access by informing the headend their buffer status. The IEEE 802.3ah standardisation group has defined the Multipoint Control Protocol (MPCP) which specifies the signalling between users and head-end enabling resource reservation [2]. The detailed execution of the reservation allocation scheme is, however, left to respective vendor to implement.

Such a simple and cost-effective time-sharing structure imposes a potential risk for poor network utilisation which therefore requires an intelligent bandwidth allocation scheme. It is thus important not to allocate too much capacity to a specific Optical Network Unit (ONU), e.g., allowing the ONU to transmit more frames than reported in the MPCP REPORT message or for a too long time relative to its and other ONU delay requirements. Such waste of capacity prolongs the circulation time of ONUs which will increase transmission delays.

In this paper, we will investigate dimensioning of EPON access networks having cost and delay constraints. Under the assumptions of the chosen structure and that the aggregate input traffic is rate limited, we derive expressions on the longest transmission delay experienced by any data frame. The maximum delay is a function of the link capacity and circulation time among 
the users, and the dimensioning problem thus becomes an optimisation problem in two variables. In this paper, we will present methods for minimising the cost of the EPON access network yielding the network with the least capacity fulfilling the delay requirements.

\section{A. Layout of Paper}

In Section II, we present the system structure and the assumptions. Here, the network components and the notation used throughout this paper is presented. In Section III, we present the operation of the system and its behaviour is mathematically formulated using the Network Calculus notation [3] [4] [5]. Performance parameters such as delay, buffer requirements and utilisation are derived for the given network structure and its operation. The dimensioning problem formulations are presented in Section IV followed by simulations verifications in Section V. In the same section, we also relax the the requirement on hard deadlines in order to increase utilisation of the system. Finally, in Section VI we summarise and conclude our results.

\section{System DEscRiption}

On the client side of the access network, a group of user network elements share an ONU as the initial access to the network, where the ONU is responsible for forwarding the user data according to a given scheduling scheme. The number of ONUs and user groups is denoted $N$ and each ONU $j$ serves $n_{j}$ different priority streams. In a practical setting, $n_{j}$ is likely to be the same for all ONUs, e.g., implementing DiffServ, but here we will be somewhat more general in our analysis. Furthermore, each ONU is controlled by a master device, the Optical Line Terminal (OLT), which controls the order in which the different ONUs may get access to the shared medium. In this paper, we will assume that each ONU will be assigned a fix duration of time $T$ during which the selected ONU may transmit frames according to the Static Priority (SP) scheme. Note that the ONUs may implement any scheduling scheme of choice in a practical setting, i.e., the functionality of an ONU is vendor specific with the only requirement that it supports the OLT signaling. Here, we will assume that all ONUs apply the SP scheduling discipline, also referred to as head-of-line non-preemption (HOL-NP), which is well covered in the literature.

The frame length of priority stream $k$ in ONU $j$ may take values in $\left[L_{1}^{j, k} \ldots L_{\text {max }}^{j, k}\right]$ according to some distribution. In this study, the frame length distribution is irrelevant, as we will investigate worst case scenarios. The data arrival process of priority stream $k$ is either actively shaped by a given regulator structure with envelope $\alpha_{j, k}(t)$ or assumed to be conforming to the above envelope, i.e., the data traffic is assumed to be conforming to the output traffic of the given regulator structure.

To motivate the fixed ONU transmission time, we will assume that all ONUs have the same channel capacity $C$. However, the time division multiplexing may result in unused capacity when the residual transmission time is shorter than the transmission time of the scheduled data frame. In case of such an event, i.e., an ONU can not transmit a high-priority frame when the residual service time is too short, it is here assumed that another frame from a lower priority flow will be transmitted instead. How this flow is selected is irrelevant to the analysis below as we only consider the worst case scenarios. However, in order to maximise the utilisation, though not particularly true to the SP discipline, such a frame could be chosen from the flow with the longest transmission time yet still not longer than the residual transmission time, given such a frame exists. This way, we will empty the transmission buffer more quickly but this action may benefit a low-priority traffic stream. An alternative method which is more true to the chosen scheduling algorithm, is to chose the frame from the flow with highest priority but with a head-of-line frame whose transmission time is not greater than the residual transmission time. In the simulations performed in this paper, we will apply the latter method.

\section{ANALYSIS}

In this section, we will investigate the maximum frame delay for either data stream. In the analysis, we will consider a specific ONU and drop the ONU index in the calculations. The analysis is identical for all ONUs since they operate independently under the assumptions above. First, we define the length of the longest data frame of priority flows $k, k+1, \ldots, n$ as

$$
L_{\max }^{[k, n]}= \begin{cases}\max \left\{L_{\max }^{k}, L_{\max }^{k+1}, \ldots, L_{\max }^{n}\right\} & , k \leq n \\ 0 & , k>n\end{cases}
$$

where $L_{\max }^{k}$ is the longest data frame of traffic flow $k$ (for valid $k$ ). To calculate the delay for the unit of data arriving at time $t$ on flow $k, d_{k}(t)$, we could determine the minimum service curve, $\beta_{k}(t)$, from which we get the delay as

$$
d_{k}(t) \leq \inf \left\{s \geq 0 ; \alpha_{k}(t) \leq \beta_{k}(t+s)\right\} .
$$

The calculation of the minimum service curve is fairly easy for the highest priority data stream even when considering the static priority scheme. However, when 
considering the vacancies in the service when another ONU is served this task becomes more complex for the lower priority data streams. Instead, we will do this the other way around, i.e., derive $d_{k}(t)$ from which we numerically get an indirect expression of the minimum service curve $\beta_{k}(t)$.

When calculating the delay, we make the following observations. At the beginning of a busy period starting at time $s$, the targeted unit, the last bit in a burst, of data of priority $k$ may need to wait for the service of a traffic source of lower priority which gets the first service. If a flow becomes active when the ONU is active, the flow may actually not transmit anything in the current window and in terms of finding the longest delay, we need only to consider this case. The amount of data to be processed is initially $L_{\max }^{[k+1, n]}$. At time $t, s<t<s+\epsilon$, all sources may begin to buffer data at full rate yielding a total amount of data $A_{k}(t)$

$$
A_{k}(t)=L_{\max }^{[k+1, n]}+\alpha_{k}(t-s)+\sum_{i=1}^{k-1} \alpha_{i}\left(d_{k}(t)+s\right)
$$

units of data that must be served prior to the targeted data unit which will finish service after a time $d_{k}(t)$. Without loss of generality, we can assume that $s=0$. Since the active ONU $j$ may have to give up transmitting

$$
L_{u}^{j}=L_{u}=\min \left\{L_{\max }^{1}, L_{\max }^{2}, \ldots, L_{\max }^{n_{j}}\right\}
$$

units of data, at the end of each transmission period, we get due to the assumptions above that the unused time is thus upper limited by $t_{u}=L_{u} / C$ since a frame of length $\leq L_{u}$ may always be transmitted when all flows are active. The delay $d_{k}(t)$ is calculated as

$$
\begin{aligned}
d_{k}(t)= & \frac{L_{\max }^{[k+1, n]}}{C}+t_{u}+\left(u_{k}(t)+1\right) \cdot N \cdot T \\
& -T+w_{k}(t)-t
\end{aligned}
$$

where $u_{k}(t)$ is the number of full transmission windows necessary to transmit $A_{k}(t)$ units of data, $T$ is the ONU transmission time and $w_{k}(t)<T-t_{u}$ is the residual amount of data transmitted in the last window. The total amount of data that is transmitted up until time $t+d_{k}(t)$, $B_{k}(t)$, is

$$
B_{k}(t)=L_{\max }^{[k+1, n]}+u_{k}(t) \cdot C \cdot\left(T-t_{u}\right)+w_{k}(t) \cdot C .
$$

By letting $w_{k}(t)=0$, we can calculate $u_{k}(t)$ by setting $A_{k}(t) \leq B_{k}(t)$. Solving for $u_{k}(t)$, i.e., finding the integer number of transmissions necessary, we can then get the $w_{k}(t)$. The total time spent on waiting in the system for the targeted data unit is thus the sum of the initial delay due to the service of the lower priority data, the initial loss of transmission opportunity, the delay due to service of other ONUs, the time spent on transmitting the data of higher priority and its own priority $k$ data. Furthermore, we know that the maximum time spent in the system for any data unit of flow $k, d_{\text {max }}^{k}$, is

$$
d_{\max }^{k} \leq \sup _{t \geq 0}\left\{d_{k}(t)\right\} .
$$

By definition we have that [5]

$$
d_{\max }^{k}=\sup _{t \geq 0}\left\{\beta_{k}^{-1}\left(\alpha_{k}(t)\right)-t\right\}
$$

from which we simply identify for some function $\nu_{k}(t)$ that

$\nu_{k}^{-1}\left(\alpha_{k}(t)\right)=\frac{L_{m a x}^{[k+1, n]}}{C}+t_{u}+\left(u_{k}(t)+1\right) \cdot N \cdot T-T+w_{k}(t)$

which is a non-decreasing function, implying that $\nu_{k}(t)$ is non-decreasing since $\alpha_{k}(t)$ is non-decreasing. Hence, $\nu_{k}(t)$ fulfills the requirements for being a minimum service curve for the observed system and flow $k$, though it may be too pessimistic. We simply assign $\nu_{k}(t)=$ $\beta_{k}(t)$ and we get

$$
\beta_{k}\left(d_{k}(t)+t\right)=\alpha_{k}(t) .
$$

At this point, we can, in the general case, numerically decide the minimum service curve and we see that, for SP, it is a function of the envelope. Furthermore, assuming that $a_{k}(0)=0$, we get from (2) that the maximum delay fulfills

$$
d_{\text {max }}^{k} \geq t_{u}+(N-1) T+L_{\max }^{[k+1, n]} / C
$$

for all rate limited priority flows $k$. Not surprisingly, we see that the delay may be decreased when shortening the transmission time $T$, though possibly at the expense of a decreased throughput.

The above calculations are based on the assumption that we are considering a well behaved system. With the chosen scheduling algorithm, the different priority streams will of course have different requirements on the system since a flow of priority $k$ may experience finite delay whilst flows with lower priorities may not. The stability criterion for the targeted ONU is based on the observation that the total amount of data put on the ONU must be less than the amount served. The capacity requirement for ONU $j, C_{j}$, must thus fulfill

$$
N \cdot T \cdot \sum_{k=1}^{n_{j}} s^{j, k}<C_{j} \cdot\left(T-t_{u}\right)=C_{j} \cdot T-L_{u}
$$

from which we get that

$$
C_{j}>N \cdot \sum_{k=1}^{n_{j}} s^{j, k}+L_{u} / T
$$


where $0<s^{j, k}<\infty$ is the sustainable data rate of priority flow $k$ in ONU $j$. Obtaining stability for the whole systems, the capacity $C$ should be greater than the maximum of all ONU capacity requirement $C_{j}$.

\section{A. Buffer Requirements}

Closely related to the delay is the maximum buffer requirement for a priority stream $k, b_{\max }^{k}$. It is easily obtained from the minimum service curve and it is calculated as [5]

$$
b_{\max }^{k} \leq \sup _{t \geq 0}\left\{\alpha_{k}(t)-\beta_{k}(t)\right\}+L_{\max }^{k}
$$

where the last term is due to the packetisation of the data stream. Since the arrival curve is non-decreasing we have that

$$
b_{\max }^{k} \geq \alpha\left(t_{0}^{k}\right)
$$

where

$$
t_{0}^{k}=\sup \left\{t \geq 0: \beta_{k}(t)=0\right\} .
$$

Having defined $t_{0}^{k}$, we may adjust (5) accordingly. However, formulating a dimensioning problem based with buffer requirement constraints is an affected problem. For this reason, we do not present such formulations and (5) will only be used for evaluation purposes.

\section{B. Throughput}

The access network is generally considered to be the bottleneck and it is important to investigate its impact on the QoS. However, when simultaneously dimensioning the access network under delay requirements, throughput concerns get secondary priority. In particular, under the assumptions of fix duration of transmission windows together with the static priority scheduling scheme used in this study, the system will generate a high throughput. Of course, putting a restriction on a lowest throughput is easily formulated in the optimisation problems seen below, though such constraint may be better motivated for traffic flows without delay constraints, especially when considering the static priority scheme.

The minimum departure process defines the least amount of data that has been transmitted at a given time $t, \gamma_{k}(t)$, and is calculated as

$$
\gamma_{k}(t)=\inf _{0 \leq s \leq t}\left\{\alpha_{k}(s)+\beta_{k}(t-s)\right\}=\left(\alpha_{k} \otimes \beta_{k}\right)(t) .
$$

Here, we have not considered the packetisation of the data flow. The minimum throughput at time $t$ for a priority data flow $k$ is thus given by $\gamma_{k}(t)$ and we are potentially interested in maximising the long run throughput, i.e., maximising $\gamma_{k}(t)$ when $t \rightarrow \infty$. This calculation is for large $t$ numerically extensive and we will for that matter not further evaluate it in the optimisation formulations below.

\section{PARAMETER OPTIMISATION}

In this section we will optimise the system performance in respect to relevant performance measures. Such measures that we are interested in here are minimum delay and buffer requirements, as well as maximum utilisation. In general, allowing a rate limiter and envelope of arbitrary type results in difficulty of explicitly solving the optimisation problem formulations below.

\section{A. Minimising Maximum Delay and Channel Capacity}

Minimising the delay for the different traffic streams is a tool for offering higher QoS. In general, we could have another objective, e.g., assigning a cost or by other means defining a penalty for longer delays and degrading QoS. The objective could thus be infinite, so we will only consider two rather straightforward optimisation objective functions.

First, we will as before assume the existence of a sustainable data rate for each priority traffic stream $k$, $0<s_{k}<\infty$, and a well behaved system with a fixed ONU capacity $C$. Our first goal is to minimise the maximum average delay, $d_{a v g, \max }$, experienced by a random data unit in a given ONU $j$. We define the objective function as

$$
d_{a v g, \max }^{k}(T)=\frac{\sum_{k=1}^{n_{j}} s^{j, k} \cdot d_{\max }^{k}(T)}{\sum_{k=1}^{n_{j}} s^{j, k}}
$$

where $d_{\max }^{k}(T)$ is given by (1) in which we let the delay be a parameter of the chosen transmission time $T$. The problem to be solved may be formulated as

$$
\begin{aligned}
\text { Minimise: } & \sum_{j=1}^{N} \frac{\sum_{k=1}^{n_{j}} s^{j, k} \cdot d_{\text {max }}^{k}(T)}{\sum_{k=1}^{n_{j}} s^{j, k}} \\
\text { Subject to: } & T>\frac{L_{u}^{j}}{C-N \cdot \sum_{k=1}^{n_{j}} s^{j, k}} \quad, j=1,2, \ldots, N
\end{aligned}
$$

In the constraint, we used (4) but also that $T$ must be greater than $L_{\max }^{[1, n]} / C$. The latter requirement is, however, always fulfilled in (4) since we assume a well behaved system. Such an optimisation problem and dimensioning formulation may be applied to construct an access network with soft delay requirements and an overall adequate performance in terms of delay. Furthermore, the above dimensioning formulation aims to minimise the overall potential maximum delay. Whether it minimises the actual delay is a matter of describing the input traffic more accurately.

In the second formulation, we will consider the delay requirements as constraints, i.e., we have upper demands 
on the frame transmission and buffering delays within the EPON access network. These delay constrains and arrival curves should here be interpreted as application specific, i.e., the delay is the typical delay value for the given service and the arrival curve is the worst case user aggregate for the application traffic. This problem then becomes more of a dimensioning problem and we need to optimise the channel capacity $C$ as well as the ONU transmission time $T$. It is thus well motivated to formulate this task as a channel capacity minimising problem. Traditionally, the capacity is treated as a costly entity and the objective function is thus well suited as a cost function, $f(C)$. The problem to solve is

$$
\begin{aligned}
\text { Minimise: } & f(C) \\
\text { Subject to: } & T>0 \\
& d_{\max }^{j, k}(T, C) \leq d^{j, k} \quad, \forall j, k
\end{aligned}
$$

where

$$
\begin{aligned}
C_{j}(T) & >N \cdot \sum_{k=1}^{n_{j}} s^{j, k}+L_{u}^{j} / T \quad, \forall j \\
C & =\max _{j=1,2, \ldots, N}\left\{C_{j}(T)\right\} \\
d_{\text {max }}^{j, k}(T, C) & =\sup _{t \geq 0}\left\{d_{k}(t, T, C)\right\}
\end{aligned}
$$

and where $d_{k}(t, T, C)$ is given by (1).

\section{VERIFICATION OF ANALYSIS}

In this section, we will apply and verify the theory just derived in Sections III and IV. The system which we aim to verify the analysis on consists of $N=16$ ONUs each carrying traffic which may take one of three priority levels (high, medium, low). The observed ONU can thus be considered as a DiffServ node. Each priority traffic stream is shaped according to a dual leaky bucket structure, with aggregate parameters $M, p, b, r$. In a more realistic setting, the aggregate arrival curve would of course be more complex, but by applying such complex envelope here would only increase the calculation load. The interpretation of the 4-tuple parameter set is the following: a single bucket with height $M<b$ bits and draining rate $p>r$ bps is used to model the burstiness of the input traffic. During a time $\tau$, the maximum amount of data that may be arrive to the ONU is limited by $M+p \cdot \tau$. After time $\tau$, the second leaky bucket limits the arrival data stream and data may only arrive at sustainable bit rate of $r$ bps. From this we see that the height of the second leaky bucket should be chosen as

$$
b+r \cdot \tau=M+p \cdot \tau \Rightarrow b=M+(p-r) \cdot \tau .
$$

The aggregate arrival curve is thus calculated as

$$
\alpha(t)=\min \{M+p \cdot t, b+r \cdot t\} .
$$

\begin{tabular}{|c|r|r|r|r|l|}
\hline Priority & $\mathrm{M}$ & $\mathrm{p}$ & $\mathrm{b}$ & $\mathrm{r}$ & $\tau$ \\
\hline 1 & $10^{5}$ & $5 \cdot 10^{6}$ & $1.5 \cdot 10^{6}$ & $10^{6}$ & 0.35 \\
\hline 2 & $10^{6}$ & $10^{6}$ & $2 \cdot 10^{6}$ & $5 \cdot 10^{5}$ & 2 \\
\hline 3 & $2 \cdot 10^{6}$ & $5 \cdot 10^{5}$ & $3 \cdot 10^{6}$ & $10^{5}$ & 2.5 \\
\hline
\end{tabular}

TABLE I

LEAKY BUCKET PARAMETERS.

The length of a data frame is in the range of 64 to 1518 bytes. The distribution of the chosen frame sizes can easily be obtained from a trace and it often shows that the uncorrelated distribution essentially is a two point distribution. Here, we will without further motivation assume that $10 \%$ of the frames are 64 bytes and the remaining $90 \%$ are 1518 bytes.

The matter of choosing appropriate bucket parameters $\left\{M_{k}, p_{k}, b_{k}, r_{k}\right\}$ for priority flow $k$, will here be resolved by simply assigning sensible numbers. We will verify previous calculations by simulating an ONU serving three priority levels with bucket parameters as defined in Table I. Given the leaky bucket parameters and frame size distributions, it is now possible to calculate the capacity and transmission time parameters as defined by the optimisation problems.

\section{A. Capacity Cost Optimisation}

In this section we will investigate the optimisation problem defined by (7). The calculations are numerically tedious and the accuracy depends on the resolution of the time parameter $t$. The optimisation problem depends on the two parameters $C$ and $T$ and there may be several combinations of the two parameters which fulfills the delay requirements. The solution to the problem is thus to find the smallest $f(C)$ such that there exists a valid $T$ fulfilling the constraints. Furthermore, due to the floor function in $d_{k}(C, T)$, an analytic expression of the optimal $C$ and $T$ is in the general case practically impossible to obtain.

For presentation, we will define the objective function simply as $f(C)=C$, i.e., we wish to minimise the capacity yet still be able to provide the delay requirements. Here, we will chose the delay requirement, for demonstration, as $d_{\max }=[0.05,0.1,0.3] s$ and ignoring i) the delay for signal propagation, $i i$ ) the guard time, and $i i i)$ the execution of the capacity allocation as described by the MPCP protocol with the motivation that the delays may be hidden in the $L_{u}$ parameter. As long as the length of the REPORT message, $L_{R E P O R T}$, is shorter than $L_{u}$, we do not need to modify the $L_{u}$ parameter. Otherwise, $L_{u} \leftarrow L_{u}+L_{\max }^{[1, n]}$ since it may be necessary to transmit one frame less in the window 


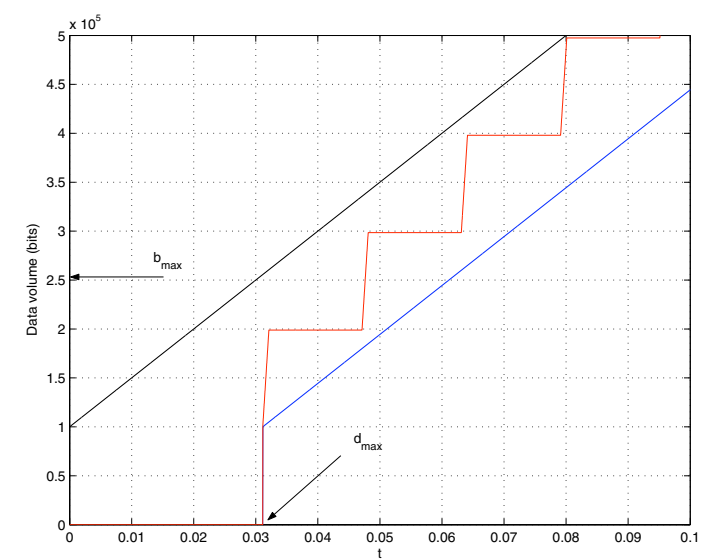

Fig. 1. Envelope (top graph), service curve (middle graph) and delayed envelope (bottom graph) for source 1 . Maximum delay is experienced by the bit served at time $t \approx 0.0320\left(d_{\max } \approx 0.0320\right.$ $\left.s, b_{\max } \leq 2.56 \cdot 10^{5} /(1518 \cdot 8)+1=22\right)$. Parameters are $C=10^{8}$, $T=0.001$ and bucket parameters as defined in Table I.

in order to fit the REPORT message. Notice that ONU signalling using the REPORT signal is not necessary under our assumptions.

Solving (7) numerically, we get the solution as shown in Table II. In the table we see that it is the medium priority traffic stream which sets the capacity requirement as it reaches the capacity constraint first. For the calculated optimal parameters, we also see that $C \cdot T / L_{\max }>62$ frames are sent in each window and the time between the transmission windows initiations is $N \cdot T=50$ ms. Furthermore, we see that the long term utilisation, $U=U_{t \rightarrow \infty}$, is rather poor for the observed structure since we do not make use of statistical multiplexing. The utilisation for the observed ONU $j, U_{j}$, is calculated as

$$
U_{j}=\frac{\sum_{k=1}^{n_{j}} s^{j, k}}{C / N} .
$$

Using the parameters declared in Table I, we get that the utilisation is upper limited by $10.6 \%$ which is horrifically low. This is obviously the result of the over provisioning and the inherent nature of the SP scheme, i.e., that delay requirements for lower priority traffic streams may require much higher capacity due to the higher priority traffic streams frequent channel access. Allowing more priority levels would likely impose even lower utilisation, hence, we make the conclusion that static priority scheduling despite its simplicity do generally not do well serving traffic streams with hard deadline requirements. In the simulations of the described systems, each traffic stream is generated by an aggregated Interrupted Poisson Process (IPP) consisting of 10 IPP sources each with squared coefficient of variation $C^{2}=100$ and average frame generation rate being $1 / 10$ of sustainable data rate

\begin{tabular}{|c|c|c|c|c|}
\hline$d_{1}$ & $d_{2}$ & $d_{3}$ & $C$ & $T$ \\
\hline 0.0474 & 0.1000 & 0.26510 & $2.4088 \cdot 10^{8}$ & $3.1280 \cdot 10^{-3}$ \\
\hline
\end{tabular}

TABLE II

ACTUAL DELAY, OPTIMAL CAPACITY AND TRANSMISSION WINDOW TIME FOR DELAY REQUIREMENT $d=[0.05,0.1,0.3]$.

\begin{tabular}{|c|c|c|c|c|}
\hline & Avg. delay & Max delay & $q_{i, \max }$ & $b_{\max }$ (calc.) \\
\hline Flow 1 & 0.02218 & 0.04711 & 17 & 28 \\
\hline Flow 2 & 0.02230 & 0.04791 & 14 & 91 \\
\hline Flow 3 & 0.02236 & 0.04809 & 6 & 176 \\
\hline
\end{tabular}

TABLE III

RESULTS OBTAINED FROM A SIMULATION USING THE

CALCULATED OPTIMAL PARAMETERS FOUND IN TABLE II. $q_{i, \max }$ IS LONGEST QUEUE LENGTH AND $b_{\max }$ IS EXPRESSED IN FULL

LENGTH FRAMES. 95\% CONFIDENCE INTERVALS OF MEANS.

as declared in Table I. The input traffic is thus assumed to conform to the output traffic of the dual-bucket structure whose input is an aggregation of several IPP processes. Any frame which is dropped due to non-conformance is not considered to be a data frame and is not retransmitted. From the simulation results we see that the average and maximum delay, especially for the second and third traffic sources, are much below the calculated maximum delay. The simulation results are of course results from, besides the bucket parameters, the chosen traffic generator and simulation time, but the results are representative for the NC's inherent pessimism. By aggregating traffic streams, as is assumed in this study, the bucket depth and draining rate (sustainable data rate) are added and, hence, the circumstances under which the



Fig. 2. Transmission time distribution for traffic flow 1 (solid), 2 (dashed) and 3 (dash-dotted) when using the calculated optimal parameters found in Table II. 
worst case scenarios occur are less probable to happen. This results in theoretical upper delay limits which are rarely experienced. In Figure 2, we can read that the capacity $C$ is more than sufficient since all three traffic flows have similar performance metrics. The difference in performance is observed in the tail of respective transmission time cumulative density function, viz., traffic streams with lower priority must wait for higher priority traffic and therefore will experience longer delay, hence, the heavier tail.

Another outcome of the vast over dimensioning, are the poorly utilised buffers. Especially traffic stream 3 is a sparing user of its buffer. This is easily verified by observing the traffic stream's delay parameters from the simulation. Since the longest experienced delay is well below the limit, then the buffer occupancy will likewise be well below its limit. Furthermore, traffic stream 3 may be very bursty though with a rather low sustainable data rate, hence, the over estimated delay and buffer size. Judging from the simulation results, it is reasonable to believe that the traffic generation may not have been particularly bursty.

\section{B. Relaxed Delay Requirements}

From the simulation results in the previous section, we see that the utilisation is unacceptably low. In this section, we intend to increase the utilisation by relaxing the delay requirements by not viewing them as hard deadlines. We will thus allow rare deadline misses. We start by inspecting the system at time $t$ in the beginning of a transmission frame. The buffer length for each traffic flow $k$ at this time is denoted $q_{k}(t)$. Assume furthermore that the last bit of information entered the buffer during the previous transmission frame. Then we have that the number of transmission frames the targeted bit of information should wait no longer than $j_{k}(C, T)$ frames given by the average waiting time $W_{k}$

$$
\begin{aligned}
W_{k} & =R+\left(j_{k}(C, T)-1\right) \cdot N \cdot T+T-t_{u} \leq d_{k} \\
& \Rightarrow j_{k}(C, T)=\left\lfloor\frac{d_{k}-R-T-L_{u} / C}{N T}+1\right\rfloor
\end{aligned}
$$

where $d_{k}$ is flow $k$ 's delay requirement, $R$ is the upper average residual waiting time and $t_{u}=L_{u} / C$ as before. Upon arrival of a randomly chosen packet must wait on average for

$$
\frac{R}{T}=\frac{1+(N-1)^{2}}{2 N}
$$

ONUs prior to achieving service since the transmission frames are fixed in duration. Requiring that $j_{k}(C, T) \geq 1$ we have from (9) and (10) that

$$
T_{k} \leq \frac{2 N}{N^{2}+2}\left(d_{k}-\frac{L_{u}}{C}\right) .
$$

The system parameter $T$ should thus not be larger than the smallest $T_{k}$. Note that we have still to decide $C$. In (10), we have assumed that $N>1$ and the tagged packet is never served in the current transmission frame. According to Little's theorem, we approximately and pessimistically get that

$$
E\left[q_{k}(t)\right]=q_{k}=p_{k} \cdot W_{k} .
$$

where $p_{k}$ is the peak data rate. From the chosen regulator structure, we can easily find the peak rate but this is perhaps not as easy for the general structure. We could however argue that, unless the peak rate is declared in any QoS negotiations, it would be reasonable to define the peak rate as the highest allowed transmission rate during a window $\tau$, e.g., calculated as

$$
p_{k}=\sup _{u>0} \frac{\alpha(u+\tau)-\alpha(u)}{\tau} .
$$

In each transmission frame, we let all traffic flows $k$ transmit $1 / j_{k}(C, T)$ of their estimated maximum queue length, i.e., all flows spread their respective buffer load evenly amongst the $j_{k}(C, T)$ transmission frames. The total capacity required, using (9), is then

$$
\begin{aligned}
C & =\frac{\sum_{k} q_{k} / j_{k}(C, T)}{T}= \\
& =N \sum_{k} p_{k}-\left(\frac{N^{2}-2}{2 N}-\frac{L_{u}}{C \cdot T}\right) \sum_{k} p_{k} / j_{k}(C, T) .
\end{aligned}
$$

Solving $C$ is now a matter of finding matching $T$ and $C$ using equations (9), (11) and (12). From (12) above, we get by letting $C \rightarrow \infty$ that

$$
C \geq \frac{N^{2}+2}{2 N} \sum_{k} p_{k} .
$$

Depending on $T$, the capacity $C$ may need to be greater than calculated in (13). This approximate analysis is also quite pessimistic and we can no longer guarantee with probability one that the data will be delivered within the requested delay. Generally, the required capacity is quite high making it probable that the utilisation is still rather low, though higher than calculated in previous sections. The average system time is more likely to be approximated by

$$
q_{k} \leq N \cdot T \cdot j_{k}(C, T) \cdot r_{k}, \quad W_{k}=R+\frac{\sum_{k} q_{k}}{C} .
$$

As an example, we could use $T=3.1280 \cdot 10^{-3}$ for the observed system according to Table II, we first see that, using (11), $T<\min _{k}\left\{2 N\left(d_{k}-L_{u} / C\right) /\left(N^{2}+2\right)\right\}$ for all $C>490 \mathrm{kbps}$, which will make further calculations easier since the calculated $C$ is less than that derived in 


\begin{tabular}{|c|c|c|c|c|}
\hline Avg. delay & Avg. delay (calc) & Max delay & $q_{i}$ & $q_{i, \max }$ \\
\hline 0.02275 & 0.02293 & 0.07314 & 3.02234 & 19 \\
\hline 0.02329 & 0.02377 & 0.16089 & 2.14626 & 16 \\
\hline 0.02358 & 0.02427 & 0.32819 & 1.19071 & 8 \\
\hline
\end{tabular}

TABLE IV

RESULTS OBTAINED FROM SIMULATION USING THE PARAMETERS $(C, T)=\left(5.97 \cdot 10^{7}, 3.1280 \cdot 10^{-3}\right)$. ONU UTILISATION IS $39.1 \%$. 95\% CONFIDENCE INTERVALS OF MEANS.

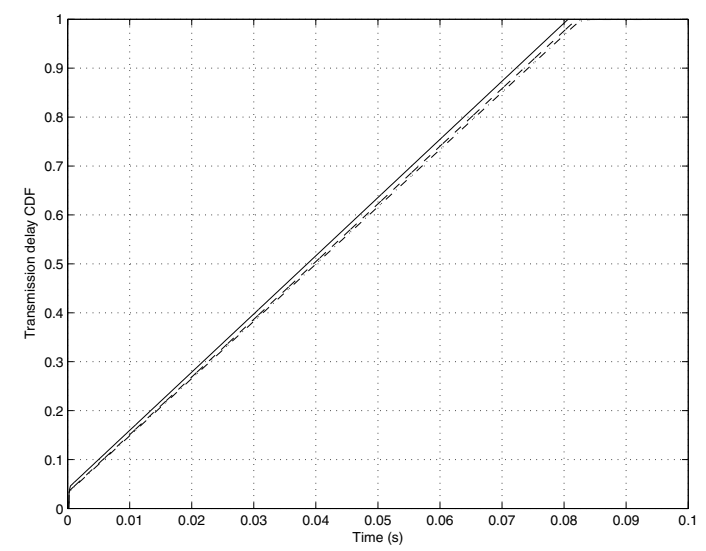

Fig. 3. Transmission time distribution for traffic flow 1 (solid), 2 (dashed) and 3 (dash-dotted) for the system parameters $(C, T)=$ $\left(5.4797 \cdot 10^{7}, 5.3473 \cdot 10^{-3}\right)$.

(13). The number of transmissions instances necessary to empty the buffer is then upper limited by

$$
\left(j_{1}, j_{2}, j_{3}\right)=(1,2,6)
$$

and we get that the delay requirement can be kept by $C=59.7$ Mbps. Thus, with less than one fourth of the capacity, we can (almost) still keep the deadline requirements for the same $T$ as seen in Table IV. Next, we could allow ourselves to change parameter $T$ as well. Finding the smallest $C$ fulfilling above requirements, we get the pair $(C, T)=\left(5.4797 \cdot 10^{7}, 5.3473 \cdot 10^{-3}\right)$. The capacity requirement is partly verified by (13). In the last simulation setup, we can see from Figure 3 that approximately $65 \%$ of the data packets are received on

\begin{tabular}{|c|c|c|c|c|}
\hline Avg. delay & Avg. delay (calc) & Max delay & $q_{i}$ & $q_{i, \max }$ \\
\hline 0.03870 & 0.03933 & 0.11393 & 5.22964 & 29 \\
\hline 0.03968 & 0.04089 & 0.25376 & 3.22928 & 20 \\
\hline 0.04011 & 0.04182 & 0.45627 & 1.33699 & 9 \\
\hline
\end{tabular}

TABLE V

RESULTS OBTAINED FROM SIMULATION USING THE RELAXED SYSTEM PARAMETER $(C, T)=\left(5.4797 \cdot 10^{7}, 5.3473 \cdot 10^{-3}\right)$. ONU UTILISATION IS $42.6 \%$. $95 \%$ CONFIDENCE INTERVALS OF MEANS. time for the first traffic flow having the strictest delay requirement. This result could arguably be too low and the reason for the low fraction of packets delivered on time is that we have not considered the bursts in the above approximation. In this paper, we have allowed packet to arrive arbitrarily close which of course is not the case in a real setting.

\section{CONCLUSIONS}

The all optical passive EPON access network is a strong candidate for providing users access to the MANs and WANs. Its low price, simple structure and high capacity enables it to be a strong candidate for the next generation access networks as today's xDSL counterpart are becoming more and more insufficient for the current applications. However, its down-scaled structure may potentially result in a poorly utilised network.

In this paper, we analyse the EPON access network under the assumptions of fixed transmission times and regulated input traffic scheduled by the Static Priority scheme. The input traffic is further assumed to be causal and shaped by regulators providing sub-additivity. With these assumptions, we have derived expressions on the longest frame delay which we have used to formulate dimensioning problems. Typically, we aim to minimise the network capacity yet still being able to fulfill the delay requirements. The delay requirements are treated as hard deadlines, i.e., delays longer than the specified limit are not tolerated. This strict rule unfortunately, but not surprisingly, results in poor network utilisation. For the specific static network structure determined by the assumptions together with the hard deadline requirements, we have seen for the provided examples that the network utilisation is less than $50 \%$. This is sufficient to motivate the use of a dynamic bandwidth allocation scheme.

\section{REFERENCES}

[1] G. Kramer and G. Pesavento, "Ethernet Passive Optical Networks (EPON):Building a Next-Generation Optical Access Network," IEEE Communications Magazine, vol. 40, no. 2, pp. 66-73, Feb 2002.

[2] "IEEE 802.3ah Ethernet in the First Mile Task Force Home Page," 2004, http://www.ieee8023.org/3/efm.

[3] R. L. Cruz, "A calculus for network delay, Part I: Network elements in isolation," IEEE Trans. Inform. Theory, vol. 37, pp. 114-131, Jan. 1991.

[4] _ - "A calculus for network delay, Part II: Network analysis," IEEE Trans. Inform. Theory, vol. 37, pp. 132-141, Jan. 1991.

[5] J.-Y. Le Boudec and P. Thiran, Network Calculus - A Theory of Deterministic Queuing Systems for the Internet. Available online at http://ica1www.epfl.ch/PS_files/NetCal.htm\#_The_book: Springer Verlag, 2001. 